\title{
CUIDADOS DE ENFERMAGEM \\ NA RESSONÂNCIA MAGNÉTICA \\ CARDÍACA
}

\author{
Washington Luiz Vieira Dias*, Thomas Pitangueira Barros** \\ Autor correspondente: Washington Luiz Vieira Dias - wlvdias@hotmail.com, \\ * Enfermeiro, Discente do curso de Pós-Graduação em Bioimagem da Escola Bahiana de Medicina e Saúde Pública, \\ Salvador-Bahia, Brasil, \\ ** Biomédico, Especialista em Bioimagem, Docente do curso de Pós-Graduação em Bioimagem da Escola Bahiana de \\ Medicina e Saúde Pública, Salvador-Bahia, Brasil.
}

\begin{abstract}
Resumo
A ressonância magnética cardíaca é um método diagnóstico que fornece informação morfofuncional em uma vasta gama de patologias do sistema cardiovascular. Por ser um exame complexo, de várias fases, torna-se relevante o papel da enfermagem na ressonância magnética cardíaca. O presente estudo tem como objetivo demonstrar as atribuições da equipe de enfermagem nos exames de ressonância magnética cardíaca como forma de garantir uma assistência específica e segura ao paciente. A metodologia utilizada foi uma revisão integrativa de caráter exploratório e do tipo descritivo utilizando as bases de dados científicos LILACS, SCIELO, MEDLINE, BDENF e PUBMED, extraindo periódicos publicados recentemente disponíveis na íntegra em português e inglês. Como conclusão o enfermeiro desempenha um papel fundamental em garantir a qualidade da assistência e a segurança do paciente em todos os aspectos relacionados ao exame de ressonância magnética cardiovascular.

Palavras-chave: Imagem por Ressonância Magnética; Gadolínio; Fármacos Cardiovasculares; Cuidados de Enfermagem.
\end{abstract}

\section{NURSING CARE IN CARDIAC MAGNETIC RESONANCE IMAGING}

\begin{abstract}
Cardiac magnetic resonance imaging is a diagnostic method that gives information on morphofunctional a wide range of pathologies of the cardiovascular system. Being a complex examination of various stages, it becomes relevant to the role of nursing in cardiac magnetic resonance. This study aims to demonstrate the functions of the nursing staff in the examination of cardiac magnetic resonance imaging as a way to guarantee a specific and safe patient care. The methodology used was an integrative review of exploratory
\end{abstract}

- Artigo submetido para avaliação em 02/10/2O14 e aceito para publicação em 17/12/2015 • 
and descriptive using scientific databases LILACS, SciELO, MEDLINE, PUBMED and BDENF, extracting recently published journals available in full in English and Portuguese. As a conclusion the nurse plays a key role in ensuring the quality of care and patient safety in all aspects related to the examination of cardiovascular magnetic resonance.

Keywords: Magnetic Resonance Imaging; Gadolinium; Cardiovascular drugs; Nursing.

\section{INTRODUÇÃO}

A ressonância magnética é um método de diagnóstico por imagem que utiliza um campo magnético uniforme e potente, para gerar imagens nos três planos anatômicos, o sagital, coronal e axial sem o reposicionamento do paciente. O mecanismo básico envolve a interação do campo magnético com as moléculas de hidrogênio (abundante no corpo humano) e pulsos de radiofrequência, que transforma-o em imagens utilizando um computador através de complexos princípios físicos e matemáticos. $(1-2)$

Atualmente a ressonância magnética tem assumido uma importância gradual no diagnóstico cardiovascular em função de seu potencial elevado de diferenciação e caracterização da anatomia e patologia cardiovascular. A ressonância magnética cardíaca (RMC) desempenha papel relevante na avaliação das diversas doenças cardiovasculares, fornecendo informações significativas sobre a anatomia, função, perfusão e viabilidade do miocárdio, além de ser considerada padrão-ouro para quantificar o volume ventricular, fração de ejeção e massa miocárdica. ${ }^{(1-3)}$

A ressonância magnética cardíaca teve um crescimento considerável nos últimos anos e agora está plenamente estabelecida em grandes centros médicos. A RMC utiliza os mesmo princípios básicos da ressonância magnética convencional, entretanto aplica técnicas e parâmetros específicos para a avaliação da anatomia, função e patologia cardiovascular. ${ }^{(1-3)}$
Segundo a Sociedade Brasileira de Cardiologia (2006) a RMC é um método diagnóstico complementar a ecocardiografia e fornece informação morfofuncional com excelente resolução espacial e temporal proporcionando um diagnóstico mais preciso. Além de reduzir a necessidade e riscos oriundos do cateterismo diagnóstico (quando a RMC é empregada previamente) e supera alguns obstáculos enfrentados na ecocardiografia. (3)

Na maioria dos exames de ressonância magnética cardíaca é utilizado um contraste paramagnético à base de gadolínio, além dos medicamentos indicados na pesquisa da avaliação da perfusão e isquemia miocárdica como o dipiridamol, adenosina e a dobutamina os quais visam melhorar a sensibilidade e especificidade do exame. ${ }^{(1-5)}$

A equipe de enfermagem deve garantir a segurança do paciente submetido ao exame de ressonância magnética cardíaca. Para tal, é essencial o amplo conhecimento acerca dos princípios de segurança da ressonância magnética e dos fármacos utilizados. ${ }^{(6-9)}$ Por se tratar de um exame complexo, de múltiplas fases, requer total atenção de toda a equipe multidisciplinar do serviço de ressonância magnética, visando a segurança, conforto e bem estar do paciente.

Desta forma, o presente estudo tem como objetivo demonstrar as atribuições da equipe de enfermagem nos exames de ressonância magnética cardíaca como forma de garantir uma assistência específica e segura ao paciente submetido à técnica. 


\section{METODOLOGIA}

Para entendimento e desenvolvimento do tema, optou-se pela pesquisa bibliográfica de caráter exploratório e do tipo descritivo.

Realizou-se um levantamento preliminar da literatura nas seguintes bases de dados: BDENF; SCIELO; LILACS; MEDLINE e PUBMED que foram acessados por meio do site do Periódicos Capes e da Biblioteca Virtual em Saúde a partir dos seguintes descritores: Imagem por Ressonância Magnética / Gadolínio / Fármacos Cardiovasculares / Cuidados de Enfermagem. Os quais foram encontrados um total de 1009 periódicos, que após a leitura e análise, foi possível selecionar 50 referências entre artigos, livros e concensos para satisfazer o objetivo proposto pelo estudo.

Foram consultados também livros de medicina e enfermagem especializados em Ressonância Magnética Cardíaca, capazes de proporcionar explicações a respeito do tema proposto.

Como critérios de inclusão foram elencados: ter sido publicado nos últimos 20 anos em periódicos nacionais e internacionais que trouxessem abordagem plena e/ou parcial do objeto de estudo, escrito em português ou inglês, e estar disponível na íntegra. Foram excluídos os artigos que não atendiam aos objetivos propostos pelo estudo.

\section{DISCUSSÃO}

O estudo do coração pela ressonância magnética originou novas responsabilidades para o enfermeiro tais como: o conhecimento sobre as patologias cardiovasculares, além dos critérios de segurança e processo de formação da imagem na ressonância magnética cardíaca. ${ }^{(10)}$

Por ser um profissional que atua diretamente nos procedimentos de diagnóstico por imagem, a resolução do COFEN $n^{\circ} 211 / 98$, normatiza a competência do enfermeiro nos serviços de diagnóstico por imagem no qual destacam entre outras funções: planejar, executar e avaliar todas as atividades de enfermagem. ${ }^{(11)}$

\section{RESSONÂNCIA MAGNÉTICA CARDÍACA X ENFERMAGEM}

A imagem por ressonância magnética aplicada em diversas áreas clínicas, conheceu, ao longo dos tempos, diversos avanços desde a sua descoberta. É uma técnica baseada na resposta específica do próton de hidrogênio (abundante no corpo humano) que, quando submetido a um campo magnético, tem a capacidade de absorver a energia de radiofrequência e convertê-la sob forma de sinal, que é captado e amplificado por bobinas localizadas na área a ser examinada..$^{(1,2)}$

As imagens de ressonância magnética podem ser ponderadas de forma a demonstrar os diferentes tipos de tecidos tais como: neuronais, fibrosos, cartilaginosos, musculares e gordurosos além de, evidenciar e analisar fluidos corpóreos como o sangue, o conteúdo biliopancreático dentre outros, através das ponderações predominantemente utilizadas em $\mathrm{T}_{1}, \mathrm{~T}_{2}$, densidade de prótons e ponderações especiais. ${ }^{(2)}$

A ressonância magnética cardiovascular teve um crescimento considerável nos últimos anos e agora está plenamente estabelecida em grandes centros médicos. Este método utiliza os mesmos princípios básicos da ressonância magnética convencional, entretanto requisita de técnicas e parâmetros específicos para a avaliação da anatomia, função e patologia cardiovascular. ${ }^{(1-5)}$

Estudos mostram que a ressonância cardíaca possui várias vantagens para o estudo de pacientes com doenças cardiovasculares os quais destacam-se: as imagens são adquiridas sem aplicação de radiação ionizante ou a administração de isótopos radioativos ou de contraste iodado; as imagens podem ser adquiridas durante o exame em qualquer plano ortogonal, superando outras modalidades diagnósticas como a ecocardiografia, cintilografia miocárdica e tomografia computadorizada; é uma modalidade de imagem flexível que 
permite a avaliação de vários parâmetros diferentes da anatomia e função cardiovascular; tem a capacidade de quantificar com boa resolução espacial e temporal medidas significativas da estrutura e/ou desempenho cardíaco. (1-5) $^{-1}$

A RMC é uma modalidade de imagem amplamente aceita, reconhecida e estabelecida na prática clínica pelo seu valor diagnóstico em uma vasta gama de doenças do sistema cardiovascular e está indicada nas cardiopatias congênitas, doença isquêmica do coração, doença arterial coronariana, cardiomiopatias / miocardite não isquêmica, doenças do pericárdio, avaliação de doença cardíaca valvular, anomalias ventriculares, angiografia pulmonar, fibrilação atrial, doença arterial periférica, doença arterial da carótida, doenças da aorta torácica, massas cardíacas, doenças congênitas, dentre outros. ${ }^{(1-5,12,13)}$

Para que a RMC forneça imagens com excelente resolução temporal e espacial faz-se necessário dispor de bobina específica para o exame, disposição correta dos eletrodos utilizados para a sincronização cardíaca (a fim de evitar interferências no traçado do ECG), do posicionamento correto do gatting respiratório (evitando assim artefatos de movimento provocados pela respiração), além do correto posicionamento do paciente. (1,2,14) $^{(1)}$

É de responsabilidade do enfermeiro realizar orientações e intervenções no preparo do paciente antes, durante e após o exame no centro de diagnóstico por imagem. Mas, para tal, deve ter pleno conhecimento técnico e científico acerca do tipo de exame, contraindicações e fármacos utilizados, além de reconhecer as possíveis complicações que possam surgir bem como realizar as ações para minimizar estes agravos. ${ }^{(6,8-10,15,16)}$

Desta forma, no período pré-estudo, o enfermeiro realiza os seguintes cuidados: avaliar o histórico do paciente e sua condição física; orientar e certificar-se da remoção completa de objetos não compatíveis com o ambiente de ressonância magnética (relógios, piercing, jóias, pagers, telefones celulares, cartões de crédito, moedas, dentre outros); orientar e certificar-se quanto ao jejum de usual de três a quatro horas (para exames de RMC); auxiliar no posicionamento do paciente na mesa de exame; realizar monitorização eletrocardiográfica com eletrodos compatíveis em ambientes de ressonância magnética posicionados geralmente na região anterior do hemitórax esquerdo, realizando tricotomia se necessário; realizar punção venosa periférica preferencialmente com cateter de calibre 18 ou $20 \mathrm{em}$ membro superior direito; orientar quanto ao procedimento a ser realizado em especial, quanto ao tempo total do exame (que pode ser longo); orientar quanto aos ruídos elevados no decorrer do exame (dispor de protetores auriculares); orientar quanto a necessidade e colaboração para a realização das apneias. Durante o exame deve avaliar os sinais vitais de acordo com o estado hemodinâmico do paciente; avaliar estado de consciência e necessidades do paciente continuamente; administrar fármacos conforme prescrição médica, atentando para as possíveis reações adversas. E por fim, após o exame, o enfermeiro deve avaliar os sinais vitais; avaliar nível de consciência; realizar o registro de enfermagem e auxiliar na transferência do paciente para sua unidade de origem. . $^{(8-10,15-18)}$

Estudos mostram que, para avaliar as contraindicações para o exame de ressonância magnética, é necessário a compreensão das questões de segurança. $O$ exame de ressonância magnética é muitas vezes descrito como " seguro" devido ao fato de que, não utiliza radiação ionizante. No entanto, existem riscos intrínsecos ao ambiente da ressonância magnética que devem ser reconhecidos e excluídos ou minimizados. ${ }^{(1,2,9)}$

Desta forma os riscos associados com a ressonância podem ser atribuídos a um e/ou uma combinação dos três principais mecanismos do sistema: o campo magnético estático, devido a fortes interações ferromagnéticas, um objeto ou dispositivo pode ser reconfigurados, movidos, girados, desalojados ou acelerados em direção ao magneto; o gradiente de campos magnéticos, podem induzir correntes elétricas em dispositivos eletricamente condutores e pode causar estimulação neuromuscular; e pulsos de radiofrequência, cujo principal 
efeito biológico é o termogênico, no qual dispositivos metálicos podem concentrar a energia de radiofrequência levando a um aquecimento local. (1-5,9,18-20)

O aspecto principal da segurança do paciente em qualquer serviço de ressonância magnética consiste em garantir a segurança magnética. Estudos mostram que os exames de ressonância magnética cardíaca são seguros, entretanto, a fim de garantir esta segurança, é extremamente importante ressaltar que, por causa do forte campo magnético, alguns objetos não podem ser submetidos ao campo devido à sua característica ferromagnética uma vez que estes podem ser atraídos pelo magneto e se comportar como projéteis. ${ }^{(9,18-20)}$

A garantia da segurança do paciente é de responsabilidade do enfermeiro. Para tal, deve-se ter pleno conhecimento dos objetos que atualmente são considerados contraindicação absoluta ou relativa para realização dos exames de ressonância magnética os quais se destacam: marca-passos; desfibriladores implantados; clipes cerebrais; bomba de insulina; dispositivos de suporte hemodinâmico (balões intra-aórticos, Swan-Ganz); dispositivos ou prótese metálica; implantes cocleares; neuroestimuladores; shunts programáveis de derivação ventricular dentre outros. ${ }^{(1-3,6-9,15-20)}$

Assim todos os serviços de ressonância magnética devem dispor de uma política de rastreamento apropriada que, na maioria delas, utilizam um questionário de segurança, que deve ser aplicado criteriosamente pela equipe de enfermagem. . $^{(15-20)}$

\section{FÁRMACOS UTILIZADOS E OS CUIDADOS DE ENFERMAGEM}

Na ressonância magnética cardíaca empreendida para testes de isquemia, são utilizados fármacos estressores tais como o dipiridamol, adenosina ou dobutamina que requerem cuidados antes, durante e após a sua administração, a fim de garantir a segurança do paciente. Portanto é dever do profissional de enfermagem ter pleno conhecimento acerca destas substâncias antes de sua administração. $(4-6,15,18)$

$\mathrm{Na}$ realização da ressonância cardíaca com estresse farmacológico o enfermeiro deve avaliar o estado hemodinâmico do paciente; verificar a pressão arterial antes do procedimento (níveis pressóricos abaixo de $90 \times 60 \mathrm{mmHg}$ contraindicam o uso dos medicamentos vasodilatadores); monitorar durante todo o exame (valores de pressão arterial periódicos antes, durante e após a indução do estresse, saturação de oxigênio, frequência cardíaca e respiratória); realizar eletrocardiograma (ECG) de 12 derivações (no início e no final do exame). Em geral os exames com estresse farmacológico com a dobutamina, adenosina ou dipiridamol são relativamente seguros, entretanto efeitos colaterais mais comuns podem surgir tais como angina, taquiarritimias e hipotensão. Desta forma, o enfermeiro deve estar apto para atender às possíveis complicações oriundas da administração destes fármacos dispondo de equipamentos e medicamentos (carrinho de parada com desfibrilador e material para reanimação) de forma a garantir a segurança do paciente. . $^{(1,18,20-24)}$

A maioria dos estudos relacionados à RMC é realizada com contraste à base de gadolínio, o que pode apresentar potenciais problemas de segurança na administração do mesmo devido aos riscos de eventos adversos. ${ }^{(1-5)}$

\section{GADOLÍNIO}

O contraste à base de gadolínio é a substância mais utilizada em ressonância magnética. $O$ gadolínio é um metal que pertence ao grupo dos lantanídeos com propriedades paramagnéticas em temperatura ambiente. Por serem altamente tóxicos, todos os meios de contraste contendo gadolínio possuem quelantes (moléculas orgânicas) na sua composição, formando um complexo mais estável e seguro, proporcionando maior tolerabilidade e excreção. ${ }^{(25-27)}$

A dose usual para estudos não vasculares é de $0,1 \mathrm{mmol} / \mathrm{kg}$ e a taxa de fluxo de injeção recomen- 
dada é de 2 a $3 \mathrm{ml} / \mathrm{s}$, sendo mais elevada para estudos de angioressonância e ressonância magnética cardíaca com doses podendo variar entre $0,2 \mathrm{a}$ $0,3 \mathrm{mmol} / \mathrm{kg}$ com um fluxo de até $5 \mathrm{ml} / \mathrm{s}$, seguida de um bolus de soro fisiológico. Assim, sua administração deve preferencialmente ser realizada em veias da fossa antecubital com dispositivos para grandes volumes (jelco 18 ou 20), para então obter imagens vasculares e da perfusão miocárdica, permitindo a completa varredura do sistema cardiovascular. $(1,5,18,21,24,25,27)$

Estudos mostram que em pacientes com função renal normal cerca de $98 \%$ destes agentes são excretados nas primeiras 24 horas da injeção, mas a utilização de altas doses ( $>0,3 \mathrm{mmol} / \mathrm{kg}$ ) em pacientes com insuficiência renal é contraindicado. (25-29)

O uso do gadolínio requer algumas precauções as quais se destacam: a necessidade em evitar seu uso em gestantes, crianças e em pacientes com insuficiência renal. Se extremamente necessário, após análise do nível de creatinina, pode-se administrar o gadolínio em pacientes com insuficiência renal com programação de hemodiálises repetidas. Ressalta-se também a necessidade do uso da pré-medicação com corticosteroide e anti-histamínicos em pacientes com história prévia de reação alérgica a contrastes de qualquer tipo ou história de alergia grave. ${ }^{(25-29)}$

Desta forma a equipe de enfermagem tem papel fundamental na identificação dos fatores de risco e prevenção de complicações relacionadas ao exame, assim faz-se necessário um planejamento adequado da assistência de enfermagem com pleno conhecimento para, junto a equipe médica, identificar e programar as intervenções mais eficazes visando a segurança do paciente. ${ }^{(15-18)}$

Os fatores de risco para reações ao gadolínio incluem uma história de alergia, insuficiência renal, asma brônquica ou reação anterior a substância à base de gadolínio. Embora existam poucos relatos na literatura sobre os malefícios do gadolínio durante a gestação ou amamentação, é recomendado evitar o uso do gadolínio no primeiro trimestre da gestação e durante a amamentação, caso seja necessário sua utilização, recomenda-se a suspensão da amamentação nas 24 horas após a administração do contraste. ${ }^{(25-31)}$

Estudos mostram que embora existam poucos relatos de reações ao gadolínio, os eventos adversos mais comuns apresentam sintomas de: sensação de calor, alteração do paladar, cefaleia transitória, urticária ou erupção cutânea, náuseas e vômitos. As reações graves e com risco de morte podem ocorrer, entretanto são extremamente raras. ${ }^{(25-31)}$

Desta forma nas reações identificadas como leve a equipe de enfermagem deve abortar imediatamente a infusão do contraste e observar o paciente por no mínimo 30 minutos. Entretanto nas reações moderadas e graves, além disso, deve acionar a equipe médica, monitorizar o paciente (frequência cardíaca e respiratória, saturação de oxigênio e pressão arterial), avaliar sinais vitais, assegurar vias aéreas e acesso venoso, além de administrar medicamentos conforme prescrição médica. $(6,15,18,25,30)$

Outra complicação durante a administração do contraste é o extravasamento. Para reduzir este risco, a equipe de enfermagem deve evitar o uso do cateter in situ com mais de 24 horas; testar a permeabilidade do cateter antes, durante e após a infusão do contraste e, em caso de dúvidas, interromper a injeção e retirá-lo; hidrolisar com solução salina após a administração do contraste; evitar administrar em acesso venoso central, caso necessário, avaliar a permeabilidade e a localização exata do cateter considerando as menores taxas de fluxo possíveis; não exceder a pressão máxima estabelecida pelos fabricantes nos dispositivos intravenosos. ${ }^{(6,15,18,32)}$

Caso ocorra o extravasamento de contraste a equipe de enfermagem deve estar preparada para atender o paciente realizando os seguintes cuidados: elevar o membro afetado acima do nível do coração; por compressa de água fria na região acometida; observar o local lesionado por no mínimo 2 horas buscando sinais de processo inflamatório; 
encaminhar para avaliação do cirurgião em extravasamentos com grandes volumes; orientar o paciente em procurar a emergência se observar mudança de sensação no membro afetado ou sinais de ulceração da pele. ${ }^{(6,15,18,32)}$

A Nefrotoxicidade induzida pelo gadolínio é rara com doses inferiores a $0,3 \mathrm{mmol} / \mathrm{kg}$. Entretanto a literatura aponta que os pacientes com insuficiência renal podem estar em risco de nefropatia induzida após a administração do contraste à base de gadolínio, podendo levar a fibrose sistêmica nefrogênica. ${ }^{(27-31)}$

A fibrose nefrogênica sistêmica (FSN) é uma doença encontrada em pacientes com insuficiência renal após a utilização do gadolínio. O mecanismo exato para o desenvolvimento de FSN permanece incerto. Os sintomas geralmente podem surgir em até quatro semanas após a exposição e é clinicamente caracterizada por edema em mãos, placas rígidas e eritematosas mais tipicamente encontradas nas extremidades, especialmente as pernas e antebraços. ${ }^{(27-31,33)}$

Devido ao risco de FSN, antes do exame de ressonância magnética, a enfermagem deve realizar uma triagem para a avaliação da função renal na maioria dos indivíduos e, em particular, os que estão no grupo de risco: pacientes idosos; pacientes com síndrome hepato-renal em associação com doença hepática grave; pessoas com história de doença ou disfunção renal; pacientes com taxas de filtração glomerular menor que $30 \mathrm{ml} / \mathrm{min}$; ou paciente com transplante renal prévio, nos quais deve-se evitar o uso do contraste. Caso seja necessário, administrar na menor dose possível, evitar a exposição repetida e programar a hemodiálise pós-procedimento de todos os pacientes com insuficiência renal (iniciando a primeira sessão até três horas após a exposição). ${ }^{(37-31,33)}$

É fundamental que todas as informações relevantes sobre o paciente sejam prontamente disponíveis antes da administração do meio de contraste para minimizar os potenciais riscos e tomar as medidas necessárias a fim de evitar uma reação adversa. ${ }^{(27-31,33)}$ Para tal, o enfermeiro deve ter pleno conhecimento acerca da farmacodinâmica e farmacocinética do gadolínio, identificando os pacientes de risco e o aparecimento de uma reação. ${ }^{(6,15,18)}$

\section{DIPIRIDAMOL}

A ressonância magnética cardíaca com estresse farmacológico, pode adicionar um valor de informação para uso na conduta terapêutica do cardiologista. A utilização do dipiridamol como agente estressor pode fornecer informações independentes para predizer eventos cardíacos em pacientes com dor torácica conhecida ou com suspeita de doença isquêmica do coração. ${ }^{(34,35)}$

O dipiridamol é um potente vasodilatador coronariano, utilizado amplamente em técnicas de produção de imagem cardíaca, indicado principalmente para a avaliação de coronariopatias isquêmicas, sendo metabolizado no fígado e são quase que totalmente excretados por via biliar (cerca de 95\%) nas fezes. ${ }^{(36,37)}$

Pesquisas revelam que a administração do dipiridamol requer uma avaliação cuidadosa, principalmente por parte da equipe de enfermagem. Neste sentido, ressalta-se que, se administrado de forma equivocada, poderá agravar o quadro clínico do paciente. ${ }^{(37-39)}$

Para se obter o efeito esperado, a equipe de enfermagem deve administrar o dipiridamol em via endovenosa inicialmente em uma concentração de 0,56 mg / kg durante 4 minutos, se bem tolerado, a concentração pode ser aumentada para dose máxima permitida em o,84 mg / kg ao longo de 6 minutos, ambos de forma contínua. Altas doses devem ser administradas num curto período de tempo ( 6 minutos) para otimizar o poder isquêmico, mas também manter um teste de esforço seguro e viável. Antes da infusão endovenosa, o dipiridamol deve ser diluído em $20 \mathrm{ml}$ numa solução de cloreto de sódio $0,9 \%$ pois, a infusão do dipiridamol não diluído pode provocar irritação local.(36-41)

Estudo realizado por Hadlich ${ }^{(41)}$ com 110 pacientes, evidenciou que o uso do dipiridamol em dose máxima tem boa tolerabilidade e segurança para 
realizar a ressonância magnética cardíaca com estresse farmacológico e afirma que todos os pacientes realizaram o exame completo, sem interrupção causada por qualquer sintoma oriundo dos efeitos do dipiridamol entretanto, ressalta cautela em pacientes cardiopatas. ${ }^{(41)}$

Porém as principais contraindicações e precauções para o uso do dipiridamol são: pacientes com história de hipersensibilidade; hipotensão arterial sistêmica; pacientes com grau elevado de bloqueio atrioventricular; estenose aórtica ou valvar e de artéria coronária; além de bradicardia sinusal (frequência menor que 45 bpm), pois estes têm chances elevadas de desenvolver uma reação adversa. Pacientes em uso de substâncias derivadas da xantina (por exemplo, cafeína, teofilina, chá, chocolate e alguns refrigerantes) devem ser orientados a suspender estas substâncias de 12-24 horas antes do exame, pois são potenciais redutores do efeito vasodilatador do dipiridamol. (1,36-39) $^{-3}$

Eventos adversos ao dipiridamol podem ocorrer tais como: dor torácica, exantemas, urticária, angioedema, laringoespasmo, broncoespasmo severo, dor abdominal, vômito, diarréia, náusea, vertigem, cefaléia, parestesia, mialgia e edema, podendo provocar também hipotensão grave e dispnéia. ${ }^{(35-41)}$

Estudos recentes ressaltam a importância de monitorar os sinais vitais durante e após 10 a 15 minutos da infusão endovenosa do dipiridamol e recomenda a realização de um eletrocardiograma utilizando pelo menos uma derivação torácica após o término do exame. Caso ocorra um evento adverso como dor torácica ou broncoespasmo, recomenda-se administrar aminofilina parenteral na tentativa de reverter o quadro clínico e o efeito do dipiridamol. (35-41)

A aminofilina é um broncodilatador que dentre suas ações age na inibição do efeitos causados pelo dipiridamol, devido a liberação de teofilina. E seu uso está contraindicado nos casos de hipersensibilidade, arritmias preexistentes, doença hepática, insuficiência cardíaca congestiva dentre outros. A dose usual é de $240 \mathrm{mg}$ ( 1 ampola de $10 \mathrm{ml}$ ) não excedendo o limite de $480 \mathrm{mg}$. Caso não alivie os sintomas de dor torácica em poucos minutos, pode-se administrar nitroglicerina sublingual. (35-39)

A equipe de enfermagem deve prestar os seguintes cuidados para a administração deste fármaco tais como: orientar o paciente a não ingerir substâncias a base de cafeína, atentar para possíveis interações medicamentosas, recomenda-se administrar de forma lenta (diluída em 10 a $20 \mathrm{ml}$ de solução salina e infundida em 1 a 2 minutos) e monitorar frequência cardíaca e respiratória. Além disso a enfermagem deve estar atenta para as possíveis reações adversas tais como hipotensão, cefaleia, convulsão, taquipneia, urticária, náusea e vômito. (35-39)

\section{DOBUTAMINA}

Nos exames de ressonância magnética cardíaca, a utilização da dobutamina, se faz necessário para identificar isquemia miocárdica, além de fornecer informações prognósticas importantes para o manejo clínico do paciente. ${ }^{(42)}$

A dobutamina é um fármaco comumente utilizado em exames de estresse cardíaco, onde produz o aumento da frequência cardíaca, melhora da condução atrioventricular e aumenta da contratilidade do miocárdico e como consequência promove a vasodilatação coronariana. A dose utilizada para se alcançar seu efeito esperado começa com $5 \mathrm{\mu g} /$ $\mathrm{kg} /$ min seguida de 10, 20, 30 até a dose máxima permitida de $40 \mu \mathrm{g} / \mathrm{kg} / \mathrm{min}$ durando cerca de no máximo cinco minutos (deverá ser administrado em bomba de infusão compatível no ambiente de ressonância magnética). Entretanto pode-se associar a administração da atropina na dose inicial de $0,25 \mathrm{mg}$ com dose máxima de 2,0 mg, ou até atingir a frequência cardíaca preconizada $(85 \%$ da frequência cardíaca máxima estabelecida para a idade). ${ }^{(43-47)}$

O tempo de ação da dobutamina geralmente ocorre em até dois minutos e sua duração aproximadamente de cinco minutos, sendo metabolizado no fígado e excretado principalmente pela urina. Este fármaco está contraindicado em pacientes 
com: taquiarritmias, feocromocitoma, estenose aórtica grave, cardiomiopatia hipertrófica obstrutiva, hipertensão arterial sistêmica grave, angina instável, miocardite, endocardite, pericardite, insuficiência cardíaca congestiva não controlada e história de hipersensibilidade a dobutamina. ${ }^{(22,36,42,43)}$

Estudos mostram que o uso da dobutamina pode desencadear várias complicações incluindo as mais graves como infarto agudo do miocárdico, taquicardia ventricular, fibrilação ventricular, ataque sistêmico transitório podendo levar até a morte. . $^{(22,36,42,43)}$

Desta forma se faz necessário realizar medidas de segurança para a utilização deste fármaco. Os estudos destacam a importância da equipe multidisciplinar, em especial a enfermagem, para identificar as possíveis complicações, visto que a administração deste fármaco é realizada de forma contínua. Além disso, o paciente deve ser monitorizado continuamente, verificando sua frequência cardíaca, saturação de oxigênio, frequência respiratória e pressão arterial.(42-47)

Os cuidados de enfermagem para administração da dobutamina são imprescindíveis para garantir a segurança do paciente os quais se destacam: administrar em bomba de infusão contínua afim de evitar doses maciças; a infusão deve preferencialmente ser administrada em veias de grande calibre; atentar para a interrupção abrupta do fármaco uma vez que pode causar hipotensão; atentar quanto ao uso de a-bloqueadores e nitratos pois podem inibir o efeito da dobutamina (deverá ser suspenso 24 horas antes do exame) e assegurar que não ocorra extravasamento, o que pode levar a uma isquemia tecidual. ${ }^{(36-39)}$

A dobutamina, além do seu valor diagnóstico encontrados na ressonância magnética cardíaca, pode fornecer informações prognósticas relevantes sobre um risco futuro de infarto do miocárdico, entretanto seu uso requer uma equipe devidamente treinada a fim de garantir o sucesso do exame e a segurança do paciente. ${ }^{(22,42,44,47)}$

\section{ADENOSINA}

Embora vários estudos têm relatado sobre a importância da RMC com estresse farmacológico utilizando a dobutamina, a maioria dos estudos em todo o mundo são realizados utilizando agentes vasodilatadores tais como adenosina ou dipiridamol. Além disso, pesquisas apontam que a adenosina tem uma melhor sensibilidade e uma tendência a maior especificidade quando comparada ao dipiridamol. ${ }^{(48,49)}$

Devido sua meia-vida ser menor que 10 segundos, a adenosina, é um agente vasodilatador usualmente seguro, de boa tolerância e de fácil controle. Seu uso geralmente leva a uma vasodilatação sistêmica, broncoconstrição, queda da pressão arterial e um aumento na frequência cardíaca. As reações adversas podem ocorrer apresentando geralmente os seguintes sintomas: opressão torácica, dispneia, síncope, cefaleia, náuseas e hiperemia da face. A equipe de enfermagem deve estar familiarizada em identificar os possíveis eventos adversos devendo prestar os seguintes cuidados: notificar o médico se os sintomas durarem mais que 1 minuto; parar imediatamente a infusão do fármaco; administrar aminofilina como antídoto (a critério médico). ${ }^{(23,36-39)}$

Para que atinja seu efeito esperado, a adenosina é geralmente administrada numa dose de 140 $\mathrm{mcg} / \mathrm{kg} / \mathrm{min}$ ao longo de 3 minutos (em bomba de infusão contínua), com dispositivos intravenosos para grandes volumes (jelco 18 ou 20 ). ${ }^{(36,39,48,49)} \mathrm{Ka}$ ramitsos ${ }^{(50)}$ demonstrou que 0 uso da adenosina em doses elevadas (até $210 \mathrm{mcg} / \mathrm{kg} / \mathrm{min}$ ) pode ser bem tolerado e seguro para os pacientes, não tendo havido qualquer complicação importante durante o estudo. ${ }^{\left({ }^{0}\right)}$ Entretanto o uso deste fármaco está contraindicado nos seguintes casos: broncoespasmo ou asma, angina instável, infarto agudo do miocárdio dentro de duas semanas do estudo, bloqueio atrioventricular de grau elevado, arritmias não controladas, estenose aórtica crítica, hipotensão persistente e hipersensibilidade a adenosina. ${ }^{(36-39,50)}$ 
Cabe ressaltar que o enfermeiro, além de ter conhecimento das contraindicações para o uso da adenosina, deve também avaliar as possíveis interações medicamentosas atentando para os fármacos como a teofilina e aminofilina que reduzem o efeito esperado da adenosina e do dipiridamol e a carbamazepina o qual potencializa o efeito da adenosina. ${ }^{(36-39)}$

Afim de garantir a segurança dos pacientes, estes devem ser monitorados continuamente avaliando a saturação de oxigênio, frequência cardíaca e pressão arterial durante todo o exame. É de responsabilidade do enfermeiro avaliar todos os pacientes e questioná-los durante e imediatamente após o término da infusão da adenosina, especialmente para a ocorrência dos seguintes sintomas: dispneia, dor torácica e outros sintomas menores (rubor, náuseas, cefaleia e síncope). ${ }^{(36-39,50)}$

\section{CONSIDERAÇÕES FINAIS}

A ressonância magnética cardíaca é, sem dúvida, o método de diagnóstico não invasivo mais eficiente para pacientes com patologias cardiovasculares. $O$ enfermeiro desempenha um papel fundamental neste serviço, proporcionando a qualidade dos cuidados ao paciente, garantindo a segurança em todos os aspectos inerentes ao exame, a fim de que se alcance o resultado clínico desejado e a satisfação do cliente.

A administração dos fármacos utilizados como o gadolínio, dipiridamol, adenosina e a dobutamina, é de responsabilidade da enfermagem. Mesmo sendo considerados seguros, é dever planejar e implementar ações que reduzam a incidência de agravos, além de garantir a segurança magnética aos pacientes.

Nos exames de RMC com o uso de agentes estressores é imprescindível que, no ambiente do setor, deve-se dispor de materiais para combater possíveis emergências e ressuscitações. Além disso, toda a equipe que participa do exame (médicos, enfermeiros, técnico de enfermagem e operadores de RM) devem ter treinamento periódicos em atendimento básico e avançado de suporte à vida em cardiologia.

É necessário atualizar continuamente o conhecimento sobre as questões de segurança, assim como na tecnologia de implantes, dispositivos, agentes de contraste e outros aspectos relacionados ao exame de ressonância magnética. Portanto, o cuidado do paciente na triagem, durante e após o exame e a avaliação precisa do risco individual, com base nas recomendações atuais, torna-se indispensável.

\section{REFERÊNCIAS}

1. Lee VS. Cardiovascular MRI: physical principles to pratical protocols. Lippincott: Williams e Wilkins; 2006.

2. Westbrook C. Manual de técnicas de ressonância magnética. $3^{\text {a }}$ ed. Rio de Janeiro: Guanabara Koogan; 2010.

3. Sociedade Brasileira de Cardiologia. Diretrizes SBC: ressonância e tomografia cardiovascular. Arq. bras. cardiol. 2006;87(3):60-100.

4. Pennell DJ, Sechtem UP, Higgins CB, Manning WJ, Pohost GM, Rademakers FE, et al. Clinical indications for cardiovascular magnetic resonance (CMR): Consensus Panel report. Eur. heart j. 2004;25(21):1940-65.

5. Hundley WG, Bluemke D, Bogaert JG, Friedrich MG, Higgins CB, Lawson MA, et al. Society for Cardiovascular Magnetic Resonance guidelines for reporting cardiovascular magnetic resonance examinations. J Cardiovasc Magn Reson. 2009;11(1):5-5.

6. Marinho RC. Manual de práticas e assistências de enfermagem no setor de diagnóstico por imagem. São Paulo: látria; 2010.

7. Leite AF, Bezerra BA, Pereira RJ, Baptista IMC. A importância da atuação do enfermeiro nos novos métodos diagnósticos não invasivos tomoressonância para coronariopatias. In: Anais do $12^{\circ}$ Encontro Latino Americano de Iniciação Científica; $8^{\circ}$ Encontro Latino Americano de Pós-Graduação; $16-17$ out. 2008; São José 
dos Campos [internet]. São José dos Campos: Universidade do Vale do Paraíba; 2008 [acesso em $2 \mathrm{O}$ out. 2O13]. 796(O2): 4. Disponível em: http://www.inicepg.univap.br/cd/INIC_2OO8/ anais/arquivosINIC/INICO796_O2_A

8. Brown CD. Improving Patient Care in the Diagnostic Imaging Department: The Role of the Nurse Navigator. J radiol nurs. 2Ol2;31(3):97100.

9. Carr MW, Grey ML. Magnetic resonance imaging" overview, risks, and safety measures. Am j nurs. 2002;102(12):26(8).

1O. Darty SN, Thomas MS, Neagle CM, Link HM, Wesley - Farrington D, Hundley WG. Cardiovascular magnetic resonance imaging: as more patients undergo these examinations, will you know what to tell them about what to expect?. Am j nurs. 2002;102(12):34(6).

11. Conselho Federal de Enfermagem. Resolução COFEN-211/1998. Dispõe sobre a atuação dos profissionais de Enfermagem que trabalham com radiação ionizante. Rio de Janeiro: COFEN; 1998 [acesso 20 maio 2013]. Disponível em: http://novo.portalcofen.gov.br/resolvocofen-2111998_4258.html

12. Hughes ML, Muthurangu V, Taylor AM. Cardiovascular MR imaging: Indications, techniques and protocols. Prog pediatr cardiol. 2010;28(1):3-10.

13. Marcu CB, Beek AM, Van Rossum AC. Clinical applications of cardiovascular magnetic resonance imaging. Can med assoc $\mathrm{j}$. 2006;175(8):911(7).

14. Nacif MS, Junior ACO, Carvalho ACP, Rochitte CE. Ressonância magnética cardíaca e seus planos anatômicos: como eu faço?. Arq. bras. cardiol. 2010;95(6):756.

15. Bianco RPR. Enfermagem no centro de diagnóstico por imagem. In: Nobrega Al, organizador. Tecnologia radiológica e diagnóstico por imagem. $4^{\mathrm{a}}$ ed. São Paulo: Difusão Editora; 2010. p. 241-268.

16. Sales OP, Oliveira CCC, Spirandelli FAP, Cândido MT. Atuação de enfermeiros em um Centro de Diagnóstico por Imagem. J Health Sci Inst. 2O10;28(4):325-328.
17. Nieto RRM. La resonancia magnética como método diagnóstico en los síndromes coronarios agudos e intervenciones de enfermería. Arch Cardiol Mex. 2007;77(supl.4):S4-254-S4-256.

18. Ishikawa WY. Preparação do paciente, segurança e manejo do contraste. In: Fernandes JL, Rochitte CE, Nomura CH, Azevedo CFF, Pinto IMF, Nacif MS et al., editores. Ressonância e tomografia cardiovascular. Barueri: Manole; 2013. p. 13-21.

19. Dill T. Contraindications to magnetic resonance imaging: non-invasive imaging. Heart BMJ. 2008;94(7):943-8.

2O. Kanal E, Barkovich AJ, Bell C, Borgstede JP, Bradley WG, Jr., Froelich JW, et al. ACR guidance document for safe MR practices: 2007. Am J Roentgenol. 2007;188(6):1447-74.

21. Fernandes JL. Técnicas básicas: perfusão. In: Fernandes JL, Rochitte CE, Nomura $\mathrm{CH}$, Azevedo CFF, Pinto IMF, Nacif MS et al., editores. Ressonância e tomografia cardiovascular. Barveri: Manole; 2013. p. 51-57.

22. Gebker R, Jahnke C, Hucko T, Manka R, Mirelis JG, Hamdan A, et al. Dobutamine stress magnetic resonance imaging for the detection of coronary artery disease in women. Heart. 2010;96(8):616-20.

23. Cerqueira MD, Verani MS, Schwaiger M, Heo J, Iskandrian AS. Safety profile of adenosine stress perfusion imaging: results from the Adenoscan Multicenter Trial Registry. J. Am. Coll. Cardiol. 1994;23(2):384-9.

24. Nagel E, Lorenz C, Baer F, Hundley WG, Wilke $\mathrm{N}$, Neubaver $\mathrm{S}$, et al. Stress cardiovascular magnetic resonance: consensus panel report. J Cardiovasc Magn Reson. 2001;3(3):267-81.

25. American College of Radiology. ACR Manual on Contrast Media. $7^{\mathrm{a}}$ ed. Reston, VA; 2010.

26. Bellin M-F, Van DM, Aart J. Extracellular gadolinium-based contrast media: An overview. Eur j radiol. 2008;66(2):160-167.

27. Nacif MS, Arai AA, Lima JAC, Bluemke DA. Gadolinium-enhanced cardiovascular magnetic resonance: administered dose in relationship to United States Food and Drug Administration (FDA) guidelines. J Cardiovasc Magn Reson. 2O12;14:18. 
28. Gauden AJ, Phal PM, Drummond KJ. MRI safety; nephrogenic systemic fibrosis and other risks. J clin neurosci. 2010;17(9):1097-1104.

29. Morcos SK, Bellin M-F, Thomsen HS, Almén T, Aspelin P, Heinz-Peer G, et al. Reducing the risk of iodine-based and MRI contrast media administration: Recommendation for a questionnaire at the time of booking. Eur $j$ radiol. 2008;66(2):225-229.

30. Elias JJ, Santos AC, Koenigkam-Santos M, Nogueira-Barbosa MH, Muglia VF. Complicações do uso intravenoso de agentes de contraste à base de gadolínio para ressonância magnética. Radiol. bras. 2008;41(4):263-267.

31. Reiter T, Ritter O, Prince MR, Nordbeck P, Wanner C, Nagel E, Baver W. Minimizing Risk of Nephrogenic systemic fibrosis in Cardiovascular Magnetic Resonance. J Cardiovasc Magn Reson. 2012;14(1):31.

32. Earhart A, Mcmahon P. Vascular access and contrast media. J infus nurs. 2011;34(2):97-105.

33. Mundim JS, Lorena SC, Abensur H, Elias RM, Moysés RMA, Castro MCM, et al. Fibrose sistêmica nefrogênica: uma complicação grave do uso do gadolínio em pacientes com insuficiência renal. AMB rev. Assoc. Med. Bras. 2009;55:220-225.

34. Bodi V, Sanchis J, Lopez-Lereu MP, Nunez J, Mainar L, Monmeneu JV, et al. Prognostic Value of Dipyridamole Stress Cardiovascular Magnetic Resonance Imaging in Patients With Known or Suspected Coronary Artery Disease. J Am Coll Cardiol. 2007;50(12):1174-1179.

35. Bodi V, Husser O, Sanchis J, Núñez J, Monmeneu JV, López-lereu MP, et al. Prognostic implications of dipyridamole cardiac MR imaging: a prospective multicenter registry. Radiology. 2012;262(1):91-100.

36. Garhart BL, Nazareno AR. Medicamentos intravenosos. 26 $6^{\mathrm{a}}$ ed. Rio de Janeiro: Elsevier; 2011. p. 43-6.

37. Clayton BD, Stock YN, Cooper SE. Farmacologia na pratica de enfermagem. $15^{\mathrm{a}}$ ed. Rio de Janeiro: Elsevier; 2O12. p. $4 \mathrm{O}$.

38. AME. Dicionário de Administração de Medicamentos na Enfermagem: 2009/2010. Rio de Janeiro: EPUB; 2009.
39. Silva ES, Viana DL. Guia de medicamentos e cuidados de enfermagem. São Paulo: Yendis; 2010.

4O. Pingitore A, Lombardi M, Scattini B, De Marchi D, Aquaro GD, Positano V, et al. Head to Head Comparison Between Perfusion and Function During Accelerated High-Dose Dipyridamole Magnetic Resonance Stress for the Detection of Coronary Artery Disease. J Am Coll Cardiol. 2008;101(1):8-14.

41. Hadlich M, Spoti M, Godoy S, Samão E, Tura BR, Braz W, et al. Segurança na realização da ressonância magnética cardíaca pela técnica combinada, utilizando dose máxima de dipiridamol para investigação de isquemia miocárdica. Rev. SOCERJ. 2007;20(3):205-211.

42. Charoenpanichkit $C$, Hundley WG. The $2 O$ year evolution of dobutamine stress cardiovascular magnetic resonance. J Cardiovasc Magn Reson. 2010;12(1):59.

43. Woo V, Lau S, Pohost GM. Safety and efficacy of early atropine injection for dobutamine stress cardiac magnetic resonance: a single center experience. J Cardiovasc Magn Reson. 2011;13(Suppl 1):127.

44. Kelle S, Hamdan A, Schnackenburg B, Köhler U, Klein C, Nagel E, Fleck E. Dobutamine stress cardiovascular magnetic resonance at 3 Tesla. J Cardiovasc Magn Reson. 2008;1O(1):44.

45. Chan C, Choi B, Jinzaki M, Kitagawa K, Tsai $\mathrm{I}-\mathrm{C}$, Yong $\mathrm{H}$, et al. $\mathrm{ASCl} 2 \mathrm{OlO}$ standardized practice protocol for cardiac magnetic resonance imaging: a report of the Asian society of cardiovascular imaging cardiac computed tomography and cardiac magnetic resonance imaging guideline working group. Int J Cardiovasc Imaging. 2010;26(2):187-2O2.

46. Kramer CM, Barkhausen J, Flamm SD, Kim RJ, Nagel E. Standardized cardiovascular magnetic resonance imaging (CMR) protocols, society for cardiovascular magnetic resonance: board of trustees task force on standardized protocols. J Cardiovasc Magn Reson. 2008;10(35):35.

47. Mamone M, Lehrke S, Lossnitzer D, Korosoglou G, Giannitsis E, Katus HA, et al. Safety and feasibility of high dose stress dobutamine mri very early after acute myocardial infarction. $J$ Cardiovasc Magn Reson. 2011;13(Suppl 1):88-88. 
48. Vasu S, Bandettini WP, Hsu L-Y, Kellman P, Leung $S$, Mancini $C$, et al. Regadenoson and adenosine are equivalent vasodilators and are superior than dipyridamole- a study of first pass quantitative perfusion cardiovascular magnetic resonance J Cardiovasc Magn Reson. 2013;15:85.

49. Paetsch I, Jahnke C, Wahl A, Gebker R, Neuss $M$, Fleck E, et al. Comparison of dobutamine stress magnetic resonance, adenosine stress magnetic resonance, and adenosine stress magnetic resonance perfusion. Circulation. 2004;110(7):835-42.

50. Karamitsos TD, Ntusi NA, Francis JM, Holloway CJ, Myerson SG, Neubaver S. Feasibility and safety of high-dose adenosine perfusion cardiovascular magnetic resonance. J Cardiovasc Magn Reson. 2010;12:66. 